\title{
Multi-Criteria Decision Analysis to Develop an Efficacy-Safety Profile of Parenteral Analgesics Used in the Treatment of Postoperative Pain
}

This article was published in the following Dove Press journal:

Journal of Pain Research

\author{
Stephan Schug $\mathbb{D}^{1,2}$ \\ Esther Pogatzki-Zahn (iD ${ }^{3}$ \\ Lawrence D Phillips ${ }^{4}$ \\ Margaret Noyes Essex (iD ${ }^{5}$ \\ Feng $\mathrm{Xia}^{5}$ \\ Alison J Reader (iD ${ }^{6}$ \\ Robert Pawinski ${ }^{6}$ \\ 'Discipline of Anaesthesiology and Pain \\ Medicine, Medical School, University of \\ Western Australia, Perth, WA, Australia; \\ ${ }^{2}$ Department of Anaesthesia and Pain \\ Medicine, Royal Perth Hospital, Perth, \\ WA, Australia; ${ }^{3}$ Department of \\ Anaesthesiology, Intensive Care Medicine \\ and Pain Therapy, University Hospital of \\ Muenster, Muenster, Germany; \\ ${ }^{4}$ Department of Management, London \\ School of Economics and Political \\ Science, London, UK; ${ }^{5}$ Pfizer Inc \\ New York, NY, USA; ${ }^{6}$ Pfizer Ltd, Walton \\ Oaks, Tadworth, UK
}

Correspondence: Margaret Noyes Essex

Tel + I 2127338018

$\mathrm{Fax}+\mathrm{I} 6464415967$

Email margaret.essex@pfizer.com
Background: Identifying the optimal treatment in an acute postoperative setting remains a challenge. Multiple analgesic options are available, but comparing outcomes is limited by a lack of head-to-head trials. In addition, decisions based on efficacy only do not take drug safety into account. In such cases, multi-criteria decision analysis (MCDA) can be utilized to quantify and compare the efficacy and safety data of various drugs.

Methodology: The efficacy-safety profiles of eight parenteral, postoperative analgesics (acetaminophen, diclofenac, ketorolac, metamizole, morphine, nefopam, parecoxib, tramadol) widely used in Europe were evaluated using an MCDA model that included 17 criteria: three for efficacy and 14 for safety. Each drug was scored on each criterion on a scale from 0 (worst) to 100 (best), according to published data and the judgment of an expert panel. A weighting process was then applied to standardize the impact of each criterion and adjust drugs' preference scores accordingly, normalizing them on the $0-100$ scale. Sensitivity analyses were also performed, including a model in which analgesic profiles were compared when opioid sparing effect was set at a zero value for all drugs.

Results: In the primary model, efficacy and safety had relative weightings of $64 \%$ and $36 \%$, respectively. Efficacy and safety criteria with the highest values were pain relief (relative weight, $29 \%$ ) and gastrointestinal effects (12\%). Parecoxib received the highest overall score (93), followed by diclofenac (80), and ketorolac (75). Morphine scored the lowest (57), due to the lack of an opioid sparing effect. When opioid sparing was given a zero rating, parecoxib remained the highest scoring analgesic (93), followed by diclofenac (80), metamizole (76), and morphine (76). Parecoxib remained the most preferred analgesic in several other sensitivity analyses.

Conclusion: This MCDA-based assessment suggests that parecoxib has the most favorable efficacy-safety profile among the assessed postoperative analgesics.

Keywords: multi-criteria decision analysis, parenteral analgesics, postoperative pain

\section{Introduction}

Various options available for postoperative pain relief have different efficacy and safety profiles. ${ }^{1}$ Recently, there has been a movement away from opioids because of their adverse effects, which can be life-threatening in the case of respiratory depression, and can also delay recovery and impair rehabilitation, in the case of nausea, vomiting, and constipation. ${ }^{2-4}$ In addition, excessive use of perioperative opioids is contributing to the ongoing "opioid epidemic" in many developed countries. 5,6 In particular, unnecessary and excessive prescribing of opioids on discharge from hospital, often by surgeons, ${ }^{7}$ may lead to subsequent long-term opioid use. ${ }^{8}$ 
The most common approach to postoperative pain relief is multimodal analgesia, ie, combining analgesics with different mechanisms or sites of action, to improve pain relief and reduce opioid use and their adverse effects. ${ }^{9}$ Reducing adverse effects leads to faster recovery and earlier discharge. ${ }^{10}$ Evidence-based treatment guidelines for postoperative pain management support the multimodal approach, but identifying the optimal treatment for each patient remains a challenge. ${ }^{11,12}$ Procedure-specific considerations for postoperative pain management complicate the choice of appropriate treatments further. ${ }^{13,14}$

Comparing efficacy-safety profiles of medicines can be challenging, ${ }^{15-17}$ because of a lack of head-to-head trials and because different clinical studies and meta-analyses use different measures of efficacy, safety, and tolerability. ${ }^{17}$ Therefore, tools that can help quantify efficacy and safety of different medicines, and present the results in a format that is easy for clinicians to understand, can be useful in treatment optimization. Multi-criteria decision analysis (MCDA) modelling in a facilitated workshop of a group of experts can be used to quantify and compare evidence-based efficacy and safety data of individual drugs, and the relevance of these data to the clinician. ${ }^{18-21}$

In this study, an MCDA model was used to compare the efficacy-safety profiles of different parenteral analgesics in the acute postoperative setting.

\section{Methods}

\section{Study Design}

The feasibility of constructing an MCDA model to determine and compare the efficacy-safety profiles of postoperative analgesics and placebo was discussed in a meeting the authors attended in October 2017. The MCDA model used in this analysis was adapted from a previously published comparison of over-the-counter analgesics. ${ }^{20}$ In a series of meetings conducted between December 2017 and July 2018, it was decided to determine and compare the efficacy-safety profiles of eight widely used parenteral (non-oral) postoperative analgesics and placebo in treatment of postoperative pain. The panel consisted of two experienced clinicians who are academic experts in postoperative pain management (SS and EP-Z), an expert in MCDA (LDP), two industrybased experts in postoperative pain medical affairs (MNE and RP), an industry-based expert in safety and tolerability (FX), and an industry-based researcher whose task was to collate efficacy and safety data (AR). The process, including drug selection, was agreed among all panel members.
The MCDA model was created in Hiview3 software (Catalyze Ltd, Winchester, Hampshire, UK). The analgesics used in this analysis were all widely available within Europe, selected on the basis of their class, and included acetaminophen (unspecified), parecoxib (non-steroidal antiinflammatory drug [NSAID], cyclooxygenase-2 [COX-2] inhibitor), metamizole, nefopam (serotonin-norepinephrine -dopamine reuptake inhibitor), ketorolac and diclofenac (NSAIDs), tramadol (atypical opioid), and morphine (opioid). Each analgesic was considered at doses that are indicated for postoperative pain management, except morphine. Since the range of morphine doses varies considerably in clinical practice, the dose of $4 \mathrm{mg}$ was identified by the panel of experts as the most appropriate for comparisons within the model.

Efficacy and safety data from the first 24 hours postsurgery were included from published randomized, double-blind studies, systematic reviews, or meta-analyses that evaluated any of the included analgesics and placebo. Where peer-reviewed, published sources were not available, data were taken from regulatory documents, including European Public Assessment Reports and Summary of Product Characteristics approved by European regulatory authorities.

\section{Criteria for Determining Efficacy-Safety Profiles}

The panel members identified and approved three efficacy criteria and 14 safety criteria to determine the efficacysafety profile of each analgesic and placebo (Figure 1 and Supplementary Table 1).

The three efficacy criteria were pain relief, defined as the proportion of patients reporting at least $50 \%$ reduction in pain intensity within 4 hours; duration of action, defined as the median time to rescue medication; and opioid-sparing effect, defined as the mean reduction (mg) of 24-hour morphine consumption. The 14 safety criteria were based on the published adverse event (AE) profiles of the analgesics and included six AEs (itchiness, nausea/vomiting, increased creatinine, hepatic effects, hypotension, and psychomimetic effects), seven serious AEs (cardiovascular thrombotic events [cardiac effects], clinically relevant respiratory depression, renal failure or clinically relevant renal impairment, serious gastrointestinal [GI] events, agranulocytosis [granulocyte concentration $<500$ cells $/ \mu \mathrm{L}$ ], serious liver dysfunction, and anaphylaxis), and the potential for toxicity 


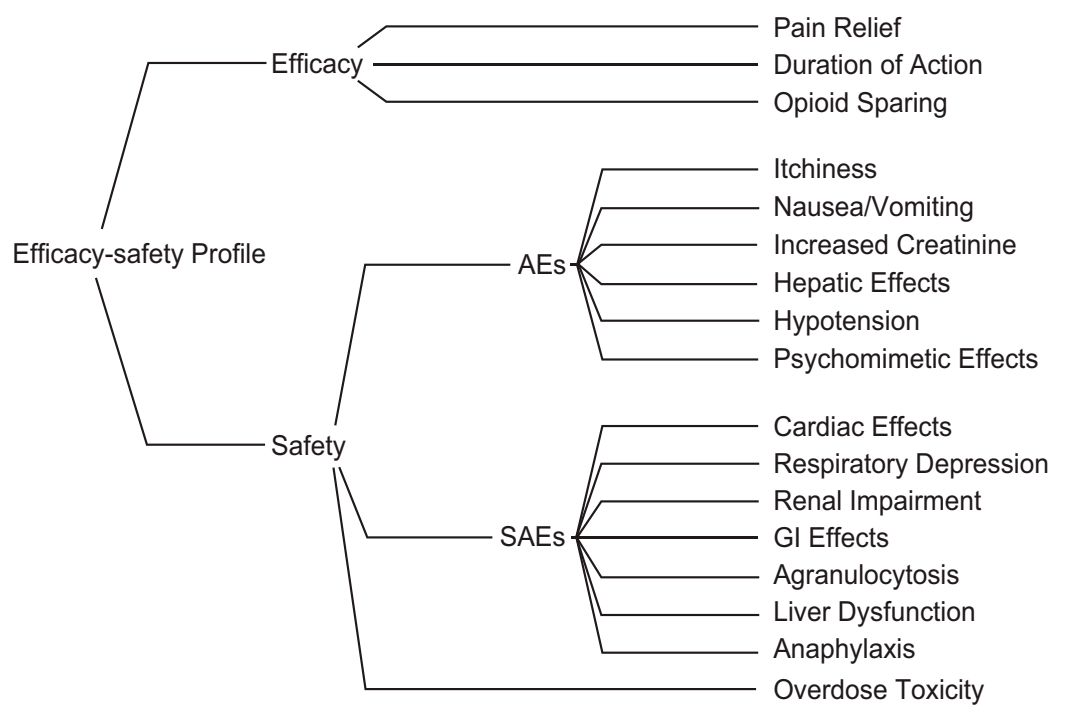

Figure I Effects tree for determining the comparative efficacy-safety balance of the analgesics and placebo. Abbreviations: AEs, adverse events; Gl, gastrointestinal; SAEs; serious adverse events.

from an accidental overdose as a separate safety category (Figure 1).

\section{Weighting of Criteria}

Because the selected efficacy and safety criteria differ in their clinical importance, the drug scores may not have the same clinical value across the criteria (ie, a score of 30 on one criterion may outweigh a score of 50 on another). To quantify this, the panel employed a "swing weighting" approach, based on participants' judgments of the clinically relevant difference between the best and worst drug on each criterion.

The process of swing weighting occurred in four steps, moving from the lowest level elements in the effects tree (ie, the individual criteria; right-most in Figure 1) to midlevel elements (groups of criteria: efficacy, AEs, serious adverse events [SAEs], safety) and then to the left-most top-level categories:

1. Low-level weighting: Relative weights in the $0-100$ range were assigned to each of the efficacy criteria; the process was repeated, separately, for the safety criteria within the AEs and the SAEs groups.

2. Mid-level safety weighting: The largest-difference criteria from the AEs and SAEs groups were compared with the Overdose Toxicity criterion; each of these criteria was assigned a weight in the $0-100$ range.

3. Top-level weighting: The largest-difference safety criterion was compared with the largest-difference efficacy criterion, and each was assigned a weight in the $0-100$ range.

4. Top- and mid-level weights were used to adjust weights of all the individual criteria, and to normalize their values on a 100-point scale, which preserves the ratios of the assessed weights.

\section{Preference Scoring of Analgesics}

For each efficacy and safety criterion, individual drugs were assigned scores ranging from 0 (the least favorable) to 100 (the most favorable). There was no score exclusivity (ie, multiple drugs could have the same score, including the score of 100). For duration of action and opioid-sparing effect, scores were determined using Hiview3 software, which linearly converted the empirical data values for each drug into values ranging from 0 to 100 . For the remaining 15 criteria ( 1 for efficacy and 14 for safety), 0-100 scores were assigned based on expert clinical opinion (SS and EP-Z), considering each drug's standing with other drugs. For example, placebo was given a score of 0 for pain relief and 100 on each of the safety criteria. A score of 50 meant that the expert panel judged that particular drug to be half-way in its performance between the most preferred drug and the least preferred drug. Of note, this scoring system, although informed by published data and clinical expertise, has an arbitrary zero point (similar to the Celsius temperature scale when compared with the Fahrenheit scale), and can be used for assessing differences in preference, but not for calculating ratios. Agreed preference 
scores or clinical data for each drug within each criterion are listed in Supplementary Table 1.

Finally, drug preference scores were adjusted by multiplying initial scores by weights for every criterion. These adjusted preference scores were used to calculate the overall preference scores for each drug, as well as score differences between morphine and each of the comparator analgesics.

\section{Sensitivity Analyses}

Additional analyses were conducted to assess (a) the value of preference scores with a zero weight placed on opioid sparing, (b) the value of efficacy scores weighted independently from safety scores, and (c) the impact of individual criteria on overall drug performance. This allowed the authors the opportunity to identify any data points that may have an overly influential impact on the model output.

\section{Statistical Analysis}

No statistical comparisons were made.

\section{Results}

Overall, the efficacy criteria were considered almost twice as impactful as the safety criteria, with assigned cumulative weights of $63.6 \%$ and $36.4 \%$, respectively. Among the individual criteria, the efficacy criterion of pain relief had the highest weighting (28.9\%); the highest weighted safety criterion was the GI effects (11.6\%) (Table 1). Details of the swing weighting process are shown in Supplementary Figure 1.

When the initial drug preference scores were adjusted using the weight of each criterion, parecoxib emerged as the most preferred analgesic, with a total score of 93, followed by diclofenac (80), and ketorolac (75) (Figure 2). Morphine had the worst efficacy-safety profile of all analgesics (total score of 57), primarily because of its inherent opioidsparing effect of zero (Figure 2).

Criterion-by-criterion differences in preference scores between parecoxib, the highest-ranked analgesic in our analysis, and morphine, the lowest-ranked, suggest appreciable advantages of parecoxib in terms of opioid sparing, respiratory depression, duration of action, and pain relief, and advantages of morphine in terms of cardiac and GI effects (Figure 3). All non-morphine drugs had an advantage over morphine in regard to opioid sparing and respiratory depression; diclofenac, ketorolac, and tramadol also had higher preference scores than morphine in regard to duration of action. For pain relief, morphine had advantage over nefopam, acetaminophen, and tramadol.

When the analysis was rerun to disregard the drugs' effect on opioid sparing, parecoxib and diclofenac remained the most preferred options, with total scores of 93 and 80, respectively, but morphine became the third most preferred option (total score of 76) (Figure 4).

In sensitivity analyses, parecoxib remained the most preferred option for a range of weights for total safety. Only

Table I Final Weights of the Criteria Comprising the MCDA Model

\begin{tabular}{|l|l|l|l|}
\hline Criterion Type & Criterion & Individual Criterion Weight (\%) & Cumulative Weight (\%) \\
\hline Efficacy & Pain relief & 28.9 & 28.9 \\
Efficacy & Opioid sparing & 23.1 & 52.0 \\
Efficacy & Duration of action & 11.6 & 63.6 \\
SAE & Gl effects & 11.6 & 75.1 \\
SAE & Respiratory depression & 9.2 & 84.4 \\
SAE & Cardiac effects & 4.6 & 89.0 \\
SAE & Renal impairment & 4.6 & 93.6 \\
SAE & Agranulocytosis & 1.2 & 94.8 \\
SAE & Liver dysfunction & 1.2 & 96.0 \\
AE & Nausea/vomiting & 1.2 & 97.1 \\
SAE & Anaphylaxis & 0.6 & 97.7 \\
Safety & Overdose toxicity & 0.6 & 98.3 \\
AE & Itchiness & 0.6 & 98.8 \\
AE & Hypotension & 0.6 & 99.4 \\
AE & Psychomimetic effects & 0.6 & 100.0 \\
AE & Increased creatinine & 0.0 & 100.0 \\
AE & Hepatic effects & 0.0 & 100.0 \\
\hline
\end{tabular}

Abbreviations: AE, adverse event; GI, gastrointestinal; MCDA, multi-criteria decision analysis; SAE, serious adverse event. 


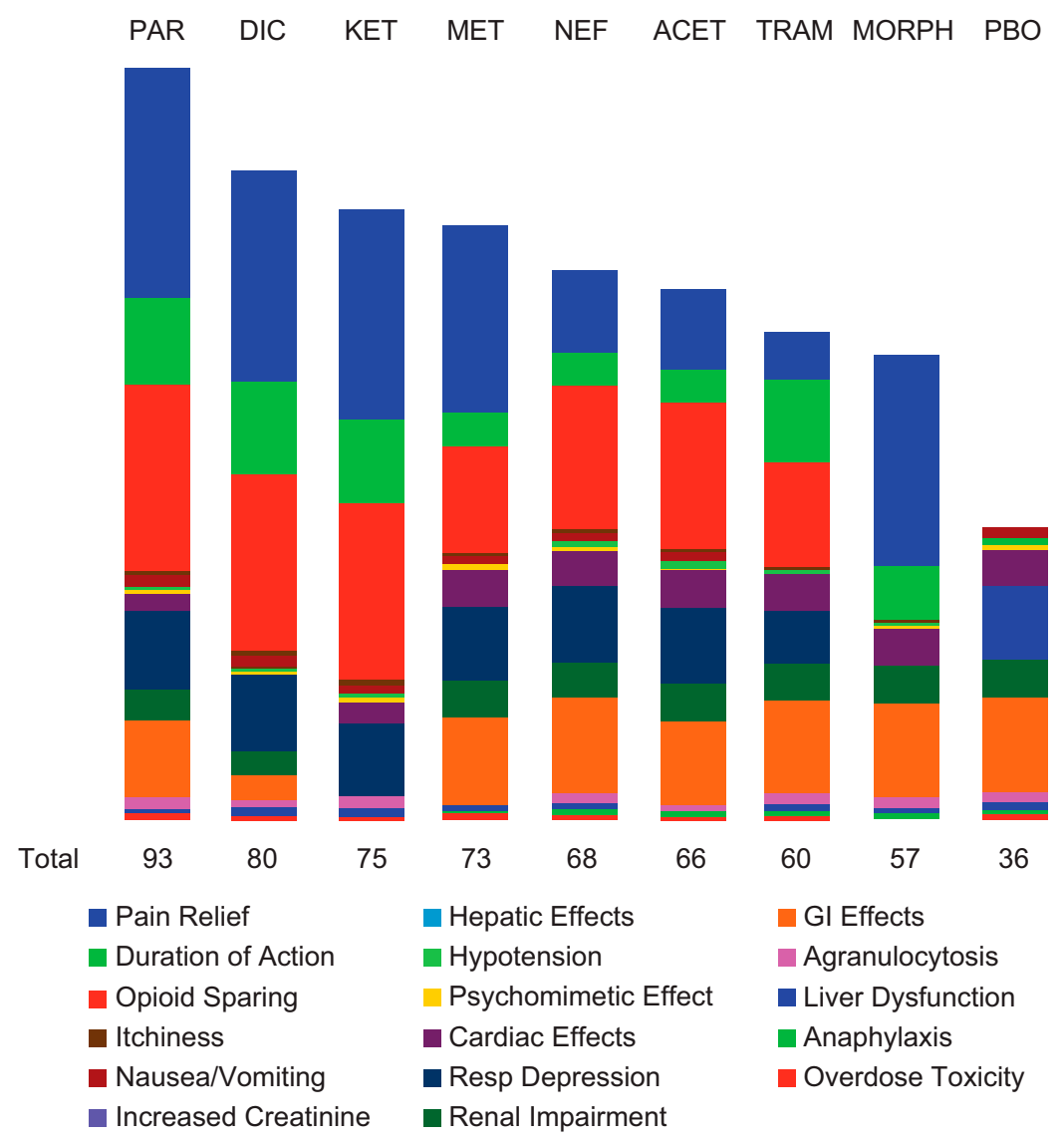

Figure 2 Adjusted preference scores of analgesics, broken down by individual efficacy and safety criteria (Primary analysis). More favorable efficacy is represented by a greater column height. More favorable safety is represented by a greater column height. The numerical values of individual efficacy and safety criteria are listed by compound in Supplementary Table I.

Abbreviations: ACET, acetaminophen; DIC, diclofenac; GI, gastrointestinal; KET, ketorolac; MET, metamizole; MORPH, morphine; NEF, nefopam; PAR, parecoxib; PBO, placebo; Resp, respiratory; TRAM, tramadol.

\begin{tabular}{|c|c|c|c|c|c|c|}
\hline & Model Order & Cum Wt & Diff & Wtd Diff & Sum & \\
\hline Benefits & Opioid Sparing & 23.1 & 100 & 23.1 & 23.1 & \\
\hline SAEs & Respiratory Depression & 9.2 & 100 & 9.2 & 32.4 & 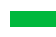 \\
\hline Benefits & Duration of Action & 11.6 & 35 & 4.0 & 36.4 & - \\
\hline Benefits & Pain Relief & 28.9 & 10 & 2.9 & 39.3 & - \\
\hline AEs & Nausea/Vomiting & 1.2 & 70 & 0.8 & 40.1 & I \\
\hline AEs & Itchiness & 0.6 & 100 & 0.6 & 40.7 & I \\
\hline Safety & Overdose Toxicity & 0.6 & 90 & 0.5 & 41.2 & I \\
\hline AEs & Psychomimetic Effect & 0.6 & 50 & 0.3 & 41.5 & 1 \\
\hline AEs & Hypotension & 0.6 & 20 & 0.1 & 41.5 & \\
\hline AEs & Increased Creatinine & 0.0 & -10 & 0.0 & 41.6 & \\
\hline AEs & Hepatic Effects & 0.0 & 0 & 0.0 & 41.6 & \\
\hline SAEs & Agranulocytosis & 1.2 & 0 & 0.0 & 41.6 & \\
\hline SAEs & Liver Dysfunction & 1.2 & 0 & 0.0 & 41.6 & \\
\hline SAEs & Renal Impairment & 4.6 & -10 & -0.5 & 41.1 & I \\
\hline SAEs & Anaphylaxis & 0.6 & -90 & -0.5 & 40.6 & I \\
\hline SAEs & Cardiac Effects & 4.6 & -50 & -2.3 & 38.3 & 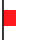 \\
\hline SAEs & GI Effects & 11.6 & -20 & -2.3 & 36.0 & 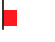 \\
\hline
\end{tabular}

Figure 3 Comparison of parecoxib versus morphine based on weighted score differences for each criterion.

Notes: Effects are shown in order of weighted difference, with sum equal to the difference in overall preference values. Positive values (green bars) indicate advantage of parecoxib and negative values (red bars) indicate advantage of morphine.

Abbreviations: AE, adverse event; Cum Wt, cumulative weight; Diff, difference; Wtd Diff, weighted difference; SAE, serious adverse event. 


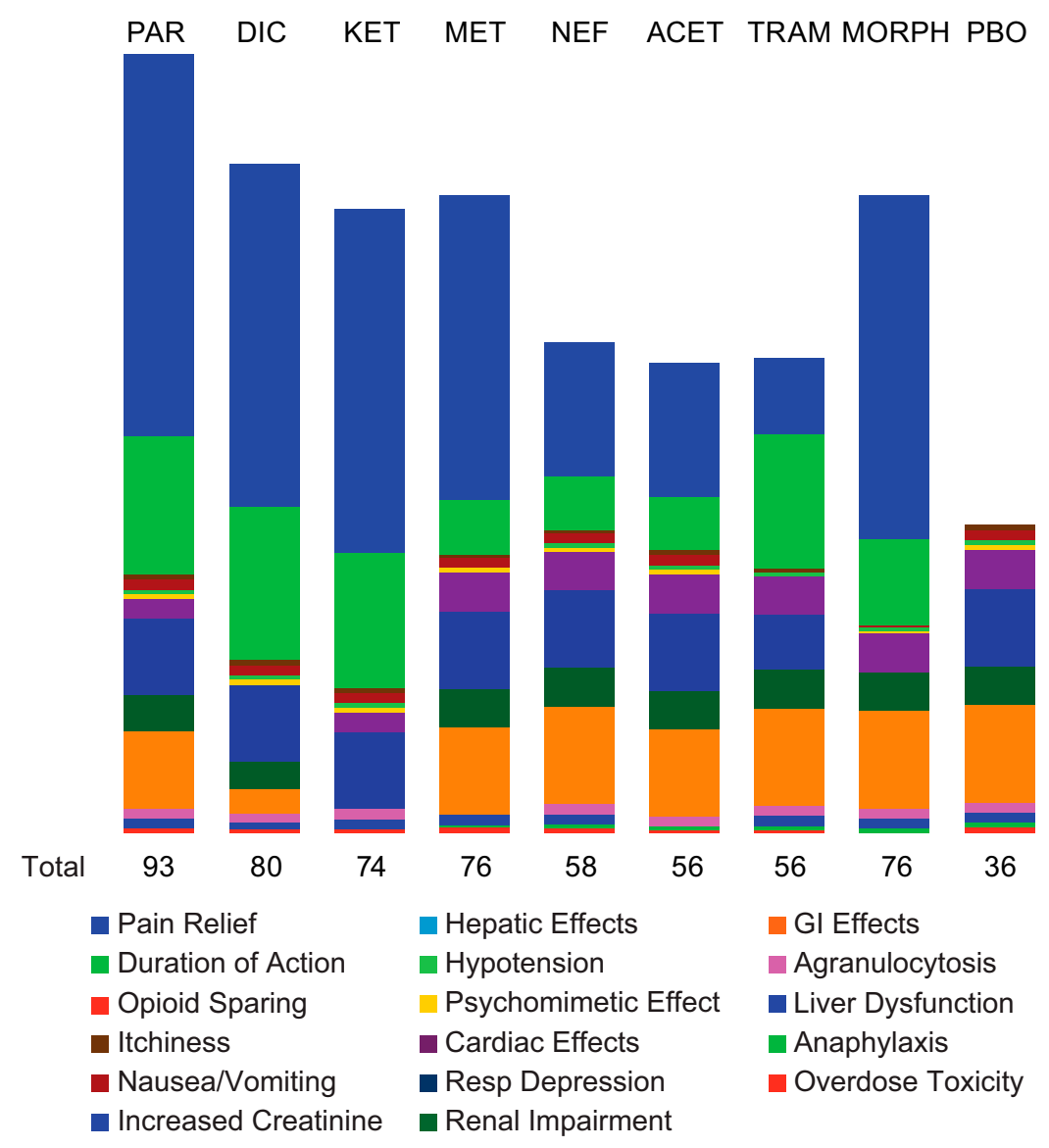

Figure 4 Adjusted preference scores of analgesics, broken down by individual efficacy and safety criteria (Sensitivity analysis: Opioid-sparing effect weight = 0). More favorable efficacy is represented by a greater column height. More favorable safety is represented by a greater column height. The numerical values of individual efficacy and safety criteria are listed by compound in Supplementary Table I.

Abbreviations: ACET, acetaminophen; DIC, diclofenac; GI, gastrointestinal; KET, ketorolac; MET, metamizole; MORPH, morphine; NEF, nefopam; PAR, parecoxib; PBO, placebo; Resp, respiratory; TRAM, tramadol.

when the weight of total safety was set above $75 \%$ did another analgesic, nefopam, become the most preferred option (Supplementary Figure 2). Parecoxib remained the most preferred option regardless of the relative weight of pain relief, with diclofenac remaining the second most preferred option for pain relief weights ranging from $10 \%$ to 100\% (Supplementary Figure 3).

When preference scores were calculated based on separately weighted values for efficacy and safety, parecoxib retained a preferred position, with an efficacy score of 98 and a safety score of 84 (Figure 5). Diclofenac and ketorolac had similar efficacy, but significantly inferior safety scores, and acetaminophen, metamizole, and nefopam were each better than parecoxib in terms of safety, but inferior in terms of efficacy (Figure 5).

\section{Discussion}

In this comparison of eight common parenteral analgesics for postoperative pain, evaluated across 17 efficacy and safety criteria, parecoxib was identified as the highestrated option in the primary analysis and in a number of sensitivity analyses. To the best of our knowledge, this is the first comparative analysis of postoperative analgesics using MCDA.

The high scores for parecoxib were largely driven by its efficacy in terms of pain relief and opioid sparing, as well as by its favorable safety profile in terms of respiratory depression and GI effects. Conversely, morphine was the least preferred option overall, mainly due to a lack of opioid sparing effect. When opioid sparing was assigned a weight of zero, morphine became the third most preferred option, highlighting the importance of opioid sparing in multimodal analgesia and the effectiveness of morphine when opioid sparing is not a consideration.

Our results are largely consistent with findings of network meta-analyses that have assessed relative efficacy of agents used for acute postoperative pain, with NSAIDs and COX-2 inhibitors among the most effective analgesics, ${ }^{22}$ 


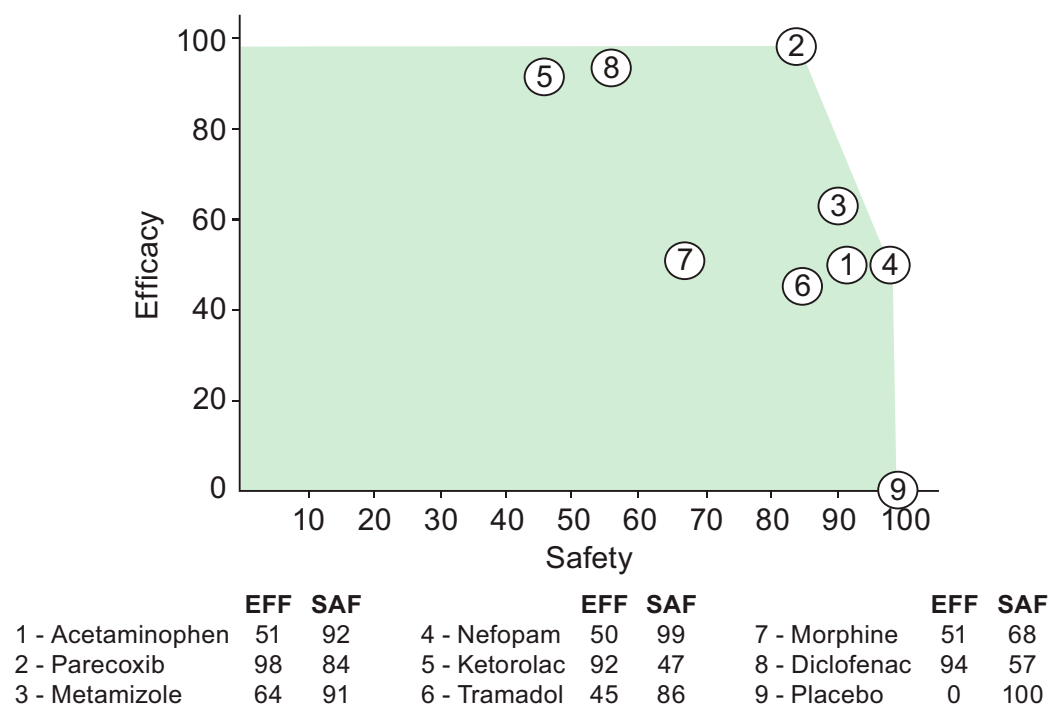

Figure 5 Preference scores of analgesics based on separately weighted efficacy and safety criteria. Abbreviations: EFF, total efficacy score; SAF, total safety score.

with an advantage over tramadol and acetaminophen, ${ }^{23}$ the second- and third-lowest ranked analgesics in our analysis. However, risk may outweigh some benefits of a drug.

The value of the MCDA model applied in our study is in its concise assessment of multiple aspects of efficacy and safety across a number of agents. In addition, it can be used to compare individual drugs across a number of criteria, which may be a valuable surrogate comparison in absence of published head-to-head trials.

It should be pointed out that our MCDA model was limited by the choice of parenteral analgesics widely available in Europe, by the types of data published for each drug, by the fact that data from different surgical procedures were combined in order to maximize the number of studies that could be included, and by the fact that it was dependent on expert opinion, which may be subjective. As such, its outcomes cannot be extrapolated to other drugs or settings.

This study is focused exclusively on the efficacy-safety balance of postoperative analgesics, which does not consider contra-indications, interactions with other drugs, or precautions. For example, the pain from different surgical procedures might respond differently to analgesics. ${ }^{14}$ Although our results are of most advantage for patients with low-risk factors for any AEs, the safety scores in Table 1 can be consulted to avoid the least safe analgesic for each side effect. For example, NSAIDs would be the least preferred for patients with high risk of cardiovascular AEs, while metamizole shows high scores for both efficacy and safety.
In conclusion, the MCDA approach we employed took into account both efficacy and safety data and drew on the expertise of experienced clinicians within the field. This assessment suggested that parecoxib, among the drugs assessed, has the most favorable efficacy-safety profile based on a wide range of criteria. Our findings may help healthcare professionals optimize treatment selection within the acute postoperative setting.

\section{Abbreviations}

AE, adverse event; COX-2, cyclooxygenase-2; GI, gastrointestinal; MCDA, multi-criteria decision analysis; NSAID, non-steroidal anti-inflammatory drug; SAE, serious adverse event.

\section{Acknowledgments}

Medical writing support was provided by Philip Matthews, PhD, CMPP, David Cope, PhD, and Vojislav Pejović, PhD, of Engage Scientific Solutions and was funded by Pfizer.

\section{Author Contributions}

All authors made substantial contributions to the concept, design, data acquisition and interpretation of the work, except AR who was involved with data acquisition. All authors, including AR, were involved in the drafting or critically revising the article, gave final approval of the version to be published; and agree to be accountable for all aspects of the work. 


\section{Funding}

This study was sponsored by Pfizer.

\section{Disclosure}

Stephan Schug was an employee of the University of Western Australia (UWA); within the last 2 years his employer, and since his retirement he himself, has received honoraria, consulting fees, and travel support from Andros Pharmaceuticals, Aspen, bioCSL, Eli Lilly, Grunenthal, Indivior, Janssen, Luye Pharma, Mundipharma, Pfizer, Pierre Fabre, Seqirus, and iX Biopharma; the travel expenses in connection with the development of this manuscript were funded by Pfizer. Esther Pogatzki-Zahn has received honoraria for lectures or advisory boards and/or travel support from Grunenthal, MSD/Merck, Fresenius, Janssen Cilag, ArcelRx, TAD Pharma, and Mundipharma. Professor Pogatzki-Zahn currently receives scientific support (unrelated to the manuscript submitted here) from the DFG, the BMBF, and the Innovative Medicines Initiative 2 Joint Undertaking under grant agreement No 777500. This Joint Undertaking receives support from the European Union's Horizon 2020 research and innovation program and EFPIA. Lawrence D. Phillips is an employee of Facilitations Limited and was a paid consultant to Pfizer in connection with this study. Margaret Noyes Essex, Alison Reader, and Feng Xia are full-time employees of Pfizer and have stock and/or stock options with Pfizer. Robert Pawinski was a paid consultant working at Pfizer at the time of the study. He has also provided consultancy services for other for-profit organizations in the pharmaceutical industry. The authors report no other conflicts of interest in this work.

\section{References}

1. Pogatzki-Zahn EM, Segelcke D, Schug SA. Postoperative pain-from mechanisms to treatment. Pain Rep. 2017;2(2):e588.

2. Boom M, Niesters M, Sarton E, Aarts L, Smith TW, Dahan A. Nonanalgesic effects of opioids: opioid-induced respiratory depression. Curr Pharm Des. 2012;18(37):5994-6004. doi:10.2174/1381 61212803582469

3. Dahan A, Aarts L, Smith TW. Incidence, reversal, and prevention of opioid-induced respiratory depression. Anesthesiology. 2010;112 (1):226-238. doi:10.1097/ALN.0b013e3181c38c25

4. Wheeler M, Oderda GM, Ashburn MA, Lipman AG. Adverse events associated with postoperative opioid analgesia: a systematic review. J Pain. 2002;3(3):159-180. doi:10.1054/jpai.2002.123652

5. Hah JM, Bateman BT, Ratliff J, Curtin C, Sun E. Chronic opioid use after surgery: implications for perioperative management in the face of the opioid epidemic. Anesth Analg. 2017;125(5):1733-1740. doi:10.1213/ANE.0000000000002458
6. Clark DJ, Schumacher MA. America's opioid epidemic: supply and demand considerations. Anesth Analg. 2017;125(5):1667-1674. doi:10.1213/ANE.0000000000002388

7. Waljee JF, Li L, Brummett CM, Englesbe MJ. Iatrogenic opioid dependence in the United States: are surgeons the gatekeepers? Ann Surg. 2017;265(4):728-730. doi:10.1097/SLA.0000000000001904

8. Brat GA, Agniel D, Beam A, et al. Postsurgical prescriptions for opioid naive patients and association with overdose and misuse: retrospective cohort study. BMJ. 2018;360:j5790. doi:10.1136/bmj.j5790

9. Buvanendran A, Kroin JS. Multimodal analgesia for controlling acute postoperative pain. Curr Opin Anaesthesiol. 2009;22(5):588-593. doi:10.1097/ACO.0b013e328330373a

10. Luo J, Min S. Postoperative pain management in the postanesthesia care unit: an update. J Pain Res. 2017;10:2687-2698. doi:10.2147/ JPR.S142889

11. Rawal N. Current issues in postoperative pain management. Eur J Anaesthesiol. 2016;33(3):160-171. doi:10.1097/ EJA.0000000000000366

12. Schug SA, Palmer GM, Scott DA, Halliwell R, Trinca J. Acute pain management: scientific evidence, fourth edition, 2015. Med J Aust. 2016;204(8):315-317. doi:10.5694/mja16.00133

13. The European Society of Regional Anaesthesia \& Pain Therapy [Internet]. PROSPECT: procedure specific postoperative pain management. Available from: https://esraeurope.org/pain-manage ment/. Accessed July 20, 2020.

14. Lee B, Schug SA, Joshi GP, Kehlet H, PROSPECT Working Group. Procedure-specific pain management (PROSPECT) - an update. Best Pract Res Clin Anaesthesiol. 2018;32(2):101-111. doi:10.1016/j. bpa.2018.06.012

15. Mussen F, Salek S, Walker S. A quantitative approach to benefit-risk assessment of medicines - part 1: the development of a new model using multi-criteria decision analysis. Pharmacoepidemiol Drug Saf. 2007;16(Suppl 1):S2-S15. doi:10.1002/pds.1435

16. Curtin F, Schulz P. Assessing the benefit: risk ratio of a drug-randomized and naturalistic evidence. Dialogues Clin Neurosci. 2011;13 (2):183-190.

17. Hughes D, Waddingham E, Mt-Isa S, et al. Recommendations for benefit-risk assessment methodologies and visual representations. Pharmacoepidemiol Drug Saf. 2016;25(3):251-262. doi:10.1002/ pds. 3958

18. Nutt DJ, King LA, Phillips LD. Independent Scientific Committee on Drugs. Drug harms in the UK: a multicriteria decision analysis. Lancet. 2010;376(9752):1558-1565. doi:10.1016/S0140-6736(10)61462-6

19. van Amsterdam J, Nutt D, Phillips L, van den Brink W. European rating of drug harms. J Psychopharmacol. 2015;29(6):655-660. doi:10.1177/0269881115581980

20. Moore A, Crossley A, Ng B, Phillips L, Sancak O, Rainsford KD. Use of multicriteria decision analysis for assessing the benefit and risk of over-the-counter analgesics. J Pharm Pharmacol. 2017;69 (10):1364-1373. doi:10.1111/jphp.12770

21. Mendoza-Sanchez J, Silva F, Rangel L, et al. Benefit, risk and cost of new oral anticoagulants and warfarin in atrial fibrillation; A multicriteria decision analysis. PLoS One. 2018;13(5):e0196361. doi:10.1371/journal.pone.0196361

22. Moore RA, Derry S, Wiffen PJ, et al. Estimating relative efficacy in acute postoperative pain: network meta-analysis is consistent with indirect comparison to placebo alone. Pain. 2018;159 (11):2234-2244. doi:10.1097/j.pain.0000000000001322

23. Martinez V, Beloeil H, Marret E, Fletcher D, Ravaud P, Trinquart L. Non-opioid analgesics in adults after major surgery: systematic review with network meta-analysis of randomized trials. $\mathrm{Br}$ J Anaesth. 2017;118(1):22-31. doi:10.1093/bja/aew391 


\section{Publish your work in this journal}

The Journal of Pain Research is an international, peer reviewed, open access, online journal that welcomes laboratory and clinical findings in the fields of pain research and the prevention and management of pain. Original research, reviews, symposium reports, hypothesis formation and commentaries are all considered for publication. The manuscript management system is completely online and includes a very quick and fair peer-review system, which is all easy to use. Visit http:// www.dovepress.com/testimonials.php to read real quotes from published authors. 